\section{Cao, Kim, Naik, Rondinelli, and Wang to chair the 2020 MRS Spring Meeting}

\author{
www.mrs.org/spring2020
}

$\mathrm{T}_{\mathrm{N}}^{\mathrm{h}}$ Meeting Chairs for the 2020 Materials Research Society (MRS) Spring Meeting are Qing Cao (University of Illinois at Urbana-Champaign, USA), Miyoung Kim (Seoul National University, South Korea), Rajesh Naik (Air Force Research Laboratory at Wright-Patterson Air Force Base, USA), James Rondinelli (Northwestern University, USA), and Hong Wang (Southern University of Science and Technology, China). The Meeting will be held April 13-17, 2020, in Phoenix, Ariz.

Qing Cao is an associate professor of materials science and engineering and of chemistry and electrical engineering at the University of Illinois at Urbana-

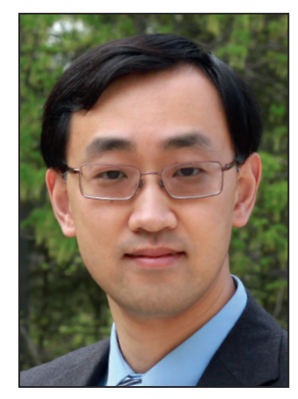

Champaign. Prior to joining the University of Illinois in 2018, Cao was a research scientist in the Department of Physical Sciences at IBM T.J. Watson Research Center. He received a $\mathrm{BSc}$ degree in chemistry from Nanjing University in 2004 and a PhD degree in materials chemistry from the University of Illinois at Urbana-Champaign in 2009.

Cao's interdisciplinary research focuses on developing functional nanomaterials for unconventional electronic systems, highperformance logic devices, and low-cost energy harvesting. He has published more than 30 research papers and is a co-inventor on 50 patents and patent applications. Cao's research has received numerous recognitions. He also made Forbes' list of "30 Under 30" in 2012 in the science category and further received the distinction of this list's "Most Influential All-Star Alumni" in 2016. MIT Technology Review listed him in 2016 as one of the top 35 global innovators under the age of 35 (TR35). In 2017, the Atlantic Council selected him as one of 21 "rising leaders and innovators around the world committed to achieving transformational change with a global impact" (Millennium Fellow).

Miyoung Kim is a professor in the Department of Materials Science and Engineering at Seoul National University, Republic of Korea. She also serves as a di-

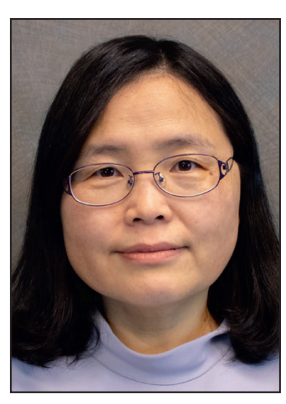

rector of the Characterization Center at the Research Institute of Advanced Materials at Seoul National University, and is on the Committee of International Cooperation for the Korean Society of Microscopy. After completing her $\mathrm{PhD}$ degree in physics from Arizona State University, with work on the charge density of $d$-electrons, Kim joined the microscopy group at Oak Ridge National Laboratory and later was a postdoctoral researcher at the University of Illinois at Chicago. She then worked at Samsung Advanced Institute of Technology on the structural characterization of high- $k$ materials and semiconductor devices.

Kim has made important contributions to the fields of resistive memory devices and energy materials using in situ transmission electron microscopy. Currently, her research focuses on retrieving atomic and electronic structures directly from scanning transmission electron microscope images and electron energy-loss spectra to explore the atomistic origin of physical properties in optical and electronic devices, catalysts, and a range of functional nanomaterials.

Rajesh Naik received his $\mathrm{PhD}$ degree in biological sciences from Carnegie Mellon University. He is currently the chief scientist of the 711th Human Performance Wing of the Air Force Research Laboratory at Wright-Patterson Air Force Base. He serves as the primary science and technology adviser to the wing commander, and he provides technical vision and strat-

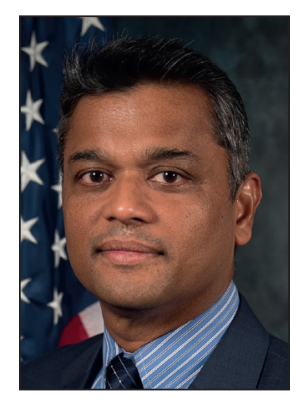

egy for the wing's USD $\$ 250 \mathrm{M}$ science and technology program in human performance and operational medicine. His research focuses on bio-nanomaterials interactions, biosensors, and engineered biomaterials. Naik has published over 300 peer-reviewed articles and he has received 13 patents. He is a Fellow of MRS, the American Institute for Medical and Biological Engineering, the Royal Society of Chemistry, SPIE, the International Society for Optics and Photonics, and the Air Force Research Laboratory. He is also an adjunct professor at the Georgia Institute of Technology (Department of Materials Science and Engineering) and at Washington University in St. Louis (Department of Mechanical Engineering and Materials Science).

James Rondinelli is the Morris E. Fine Junior Professor in Materials and Manufacturing at Northwestern University (NU), in the Materials Science

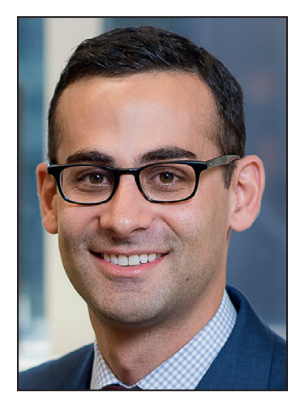
and Engineering (MSE) Department and Applied Physics Program, where he leads the Materials Theory and Design Group. His interests include electronic structure theory and first-principles design of functional inorganic materials using picoscale structure-property relationships. He received a BS degree in MSE from NU (2006) and a PhD degree in materials from the University of California, Santa Barbara (2010).

Rondinelli is a 2018 Kavli Frontiers of Science Fellow and the 2017 MRS Outstanding Young Investigator. He was named an Emerging Young Investigator by the Royal Society of Chemistry (2016) and the American Chemical Society (2014). Rondinelli has (co)authored more than 130 peer-reviewed publications and is a member of MRS, the American Physical Society, American 
Chemical Society, The Minerals, Metals \& Materials Society, The American Ceramic Society, and American Society for Engineering Education. He serves as an editorial board member of the Journal of Physics: Condensed Matter and NPJ Computational Materials. Rondinelli is also chair of the Argonne Center for Nanoscale Materials Users' Executive Committee (2016-2019).

Hong Wang is currently a chair professor and vice dean of the College of Engineering at the Southern University of Science and Technology, China. Previously, she was a professor at Xi'an Jiaotong University. She received her

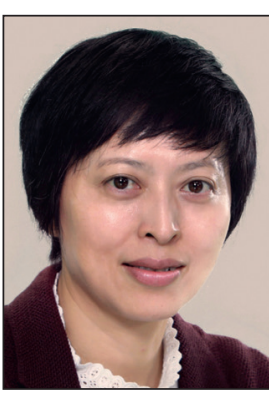

$\mathrm{PhD}$ degree in electronics materials and devices from Xi'an Jiaotong University. Wang's main research interests include dielectric materials, multifunctional composites, and dielectric measurements. She has received many honors, including the Distinguished Young Scholars Award from the National Natural Science Foundation of China (2010), Chang Jiang Scholars Award from the Ministry of
Education of China (2011), and the Scientific Innovation Leaders of the National 10000 Talents Program (2016).

Wang has (co)-authored more than 240 papers, 28 patents, and has delivered more than 50 invited talks at international conferences. She is the chair of the Asian Electroceramic Association (AECA). She is a member of the IEEE Ferroelectrics Committee. She also serves as an associate editor for IEEE Transactions on Ultrasonics, Ferroelectrics, and Frequency Control, and is an editorial board member for the Journal of Advanced Ceramics, the Journal of the Chinese Ceramic Society, and IET Nanodielectrics.

\section{4th International Symposium on Macrocyclic and Supramolecular Chemistry} will be held in Italy https://ismsc2019.eu

The 14th International Symposium

1 on Macrocyclic and Supramolecular Chemistry (ISMSC2019) will be held in Lecce, Italy, on June 2-6, 2019.

ISMSC2019 will provide a forum to discuss all aspects of macrocyclic and supramolecular chemistry and also topics on materials and nanoscience. This symposium will be a unique opportunity for researchers to brainstorm and promote cross-disciplinary collaborations that will have an impact on their research and related fields of applications (e.g., biomedical engineering, food science, energy storage, biosensing). It will also offer networking opportunities among peers, recognized leaders in the field, young scientists, and students. The program will include lectures from the 2016 Nobel laureates in Chemistry, from the 2019 IzattChristensen Award, Cram-Lehn-Pedersen Prize, and Sessler Early Career Researcher
Prize winners. ISMSC2019 will also host the Natural Product Reports Emerging Investigator Lectureship. Lectures, flash presentations, and poster presentations will also be selected from submissions. Scholarships covering registration fees are available for select young scientists.

Abstract submissions are due January 15 , and early bird registration ends February 15. Visit https://ismsc2019.eu for more details.

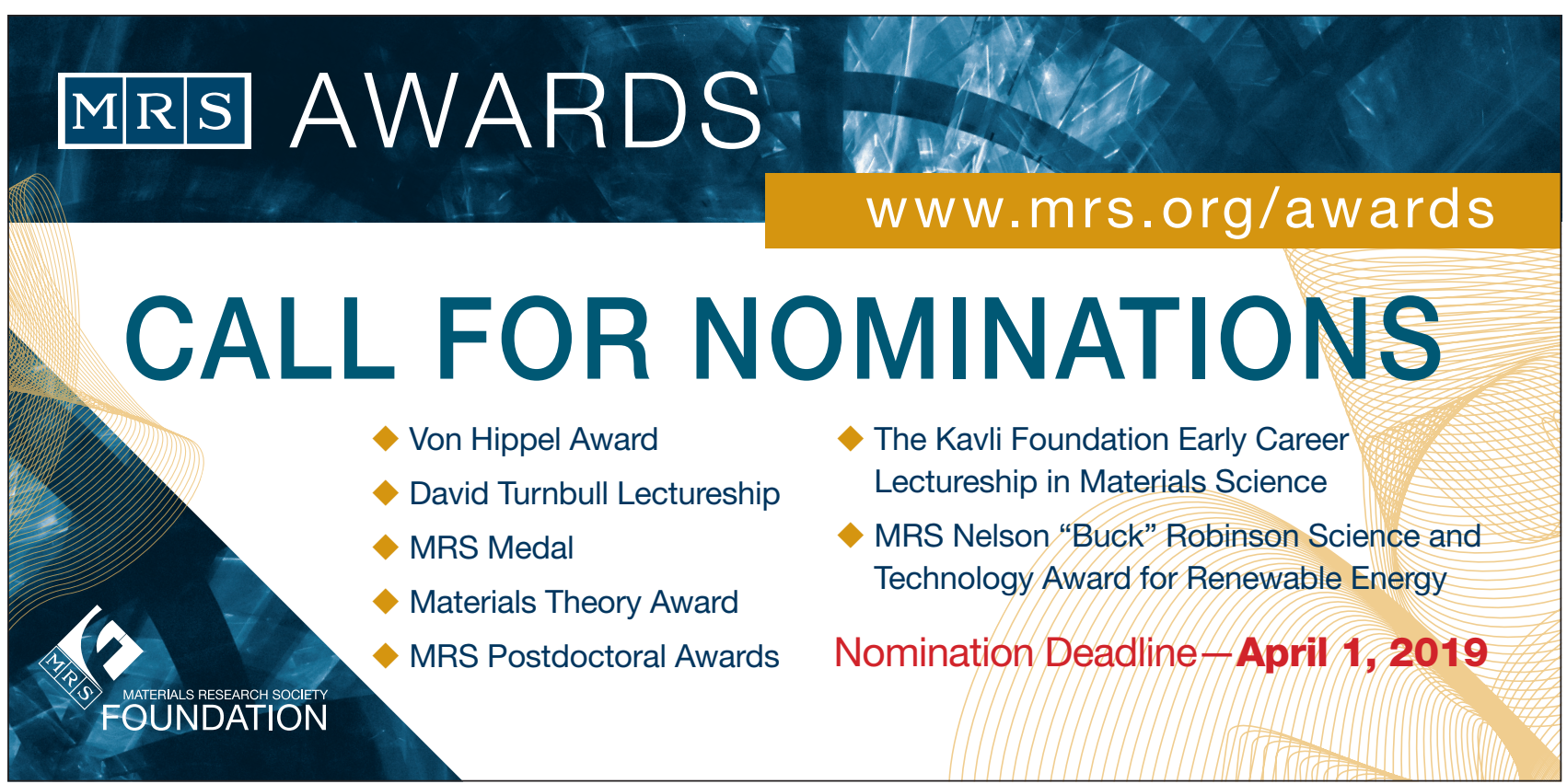

